\title{
The impact of early percutaneous tracheotomy on reduction of the incidence of ventilator associated pneumonia and the course and outcome of ICU patients
}

ZORAN KARLOVIĆ ${ }^{1}$, DAJANA VLADIĆ ${ }^{1}$, MATEO PERIĆ ${ }^{1}$, MIRKO MIHALJ ${ }^{1}$, ŽELJKA ZADRO ${ }^{1}$, VIŠNJA MAJERIĆ-KOGLER

${ }^{1}$ Department of Anesthesia, Reanimation and Intensive Care, University Clinical Hospital Mostar, Mostar, Bosnia and Hercegovina

${ }^{2}$ University of Zagreb, School of medicine

Corresponding author:

Mateo Perić

Department of Anesthesia, Reanimation and Intensive care

University Clinical Hospital Mostar

Mostar, Bosnia and Hercegovina

Phone: +38763391680

Fax: +38736336201

E-mail:mateo.peric@gmail.com

\section{ABSTRACT}

The aim of the study. The primary objective of this study was to determine the differences in the incidence of respiratory infections and septic episodes in patients who underwent early percutaneous tracheotomy (ET) and in patients who underwent translaryngeal intubation i.e late tracheotomy (LT). Secondary objectives were to determine the differences in the early mortality of patients, duration of mechanical ventilation and length of Intensive care unit (ICU) stay.

Materials and methods. The study included 72 surgical and trauma patients older than 18 years of age, treated at the ICU of the University Clinical Hospital Mostar who had undergone translaryngeal intubation and were mechanically ventilated for at least 48 hours. The basic criterion for inclusion in the study was expected duration of mechanical ventilation of at least 14 days. Forty-eight hours after enrollment, patients were randomly divided into two groups. The first group of patients underwent ET after 2-4 days of mechanical ventilation; the second group underwent LT if they exhibited longer episodes of hypoxemia after 15 days.

Results. The ET group of patients spent less time in mechanical ventilation and ICU. The ET group had a lower rate of VAS pneumonia $(p=0.137)$, sepsis episodes $(\mathrm{p}=0.029)$ and mortality rate $(\mathrm{p}=0.056)$.

Conclusion. The results of our study support ET being performed 2-4 days from the start of mechanical ventilation. Despite a lack of power, we found significant benefits of ET regarding the incidence of pneumonia, sepsis, hospital mortality, duration of mechanical ventilation and length of ICU stay

Key words: tracheotomy, mechanical ventilation, intensive care unit, ventilator-associated pneumonia, treatment outcome, complications

\section{INTRODUCTION}

The optimal method of treating the airways and implementing the process of mechanical ventilation in patients who are unable to breathe spontaneously, has been the object of clinicians' interest for almost a hundred years. (1) In comparison with translaryngeal intubation, the benefits of early percutaneous tracheotomy (ET) include: prevention of serious injury to the larynx, greater airway safety, decreased airway resistance, facilitated pulmonary hygiene, and oral cavity care which can reduce the incidence of developing lung infections, help to facilitate the weaning process of mechanical ventilation, and greater mobility and comfort of patients. (2)

Although the rate of tracheotomy procedures, in particular percutaneous tracheotomy, has, according to the literature, increased by $200 \%$ in recent years, the analysis of major databases still continues to show an unbalanced approach in regards to the time required to perform tracheotomy procedures, early or late, and the conflicting and inconsistent results relating to the incidence of nosocomial infection, cardiac complications, duration of treatment, dura- tion of mechanical ventilation, and survival rate of patients. (3)

Adesina et al. point out the reduced number of days on the ventilator and shorter treatment in the Intensive care unit (ICU) as benefits of ET; however, their study did not report a decrease in hospital mortality rate. (4) Shorter treatment in ICUs is cited in other studies as well, (5-7) while Mahafza et al. and Rumbak et al. reported a decrease in mortality rate as a benefit of ET too. $(8,9)$ In a study by Brook et al., the reduction of hospital costs is cited to be one of the benefits, which is not to be disregarded. (10)

The majority of previous studies is retrospective, time spent performing ET in the cited studies was too big (two to ten days), while non-homogeneous groups of patients made it difficult to interpret the results. Most studies did not precisely explain how randomization of patients was done, or the criteria according to which the patients were assessed for expected duration of mechanical ventilation.

The primary objective of this study was to determine the differences in the incidence of respiratory infections and septic episodes in patients who underwent ET and in patients who underwent translaryngeal intubation i.e. LT. Secondary objectives were to determine the differences in early mortality of patients, duration of mechanical ventilation and length of ICU stay.

\section{MATERIALS AND METHODS}

The study included 72 surgical and trauma patients older than 18 years of age, treated 
at the ICU of the University Clinical Hospital Mostar who underwent translaryngeal intubation and were mechanically ventilated for at least 48 hours.

The study was approved by the Ethics Committee of the University Clinical Hospital Mostar (3108/13,29.05.2013).

The basic criterion for inclusion in the study was the expected duration of mechanical ventilation for at least 14 days based on the diagnosis of patients SOFA score $>5$, APACHE II scores $>10, \mathrm{PaO} 2$ $\leq 60 \mathrm{mmHg}$ with $\mathrm{FiO} 20.5$ and PEEP of at least $8 \mathrm{~cm} \mathrm{H} 2 \mathrm{O}$. Patients who had already undergone tracheotomy, patients with a history of anatomical deformity of the neck, or suffering from hematologic malignancies and patients who were diagnosed with a respiratory infection within the first $48 \mathrm{~h}$ of mechanical ventilation were all excluded from the study. Due to the specific condition of patients, informed written consent for participation in the study was signed by a member of the patient's immediate family.

Forty-eight hours after enrollment, patients were randomly divided into two groups. The first group of patients underwent early tracheotomy (ET) after 2-4 days of mechanical ventilation, while the patients in the second group were ventilated through the endotracheal tube. The patients in the second group underwent late tracheotomy (LT) if they exhibited longer episodes of hypoxemia after 15 days, identified as $\mathrm{PaO} 2<60 \mathrm{mmHg}$ with $\mathrm{FiO} 20.6$ and $\mathrm{PEEP}=8 \mathrm{~cm}$.

Percutaneous dilatational tracheotomy was performed in the ICU. Over the treatment course, all complications related to tracheotomy were noted and classified as:

1. intraoperative: minor or significant bleeding, difficult tracheotomy tube placement (i.e., requiring $>2$ attempts at insertion during primary placement procedure), hypoxia (oxygen saturation of $<90 \%$ for $60 \mathrm{sec}$ ) and arrhythmia.

2. post-operative: inflammatory changes around the stoma, pneumothorax, subcutaneous emphysema, tracheoesophageal fistula, cannula displacement, and the need for cannula replacement.

All patients were assessed according to the APACHE II score upon admittance to the ICU, while SOFA scores were determined $48 \mathrm{~h}$ after the start of ventilation. All patients were mechanically ventilated with ventilator settings (tidal volume $6-7 \mathrm{ml} / \mathrm{kg}$, peak pressure $<35 \mathrm{~cm}, \mathrm{PEEP}>5 \mathrm{~cm} \mathrm{H} 2 \mathrm{O}$ ) in accordance with good clinical practice. The process of weaning from the ventilator was done according to the algorithm of gradually reducing ventilator support dur- ing pressure support ventilation (PSV).

At the start of the study, every patient was classified according to: age, gender, primary diagnosis, type of surgical procedure (abdominal or urological, neurological, vascular), emergency or elective surgical procedure. In addition a complete hematological and biochemical analysis and surveillance cultures of nasal and rectum swabs were performed. All patients underwent qualitative and quantitative analysis of bronchial secretions using the bronchoalveolar lavage technique 48 hours after the start of mechanical ventilation. Ventilator associated pneumonia (VAP) was diagnosed based on standardized criteria: body temperature $<38$ or $>36$, purulent bronchial secretions, new lung infiltrate observed on X-ray, qualitative and quantitative microbiological analysis of the bronchial secretion obtained by bronchoalveolar lavage and laboratory diagnostics (leucocyte count, C-reactive protein, etc). (11)

Septic episodes were diagnosed based on: clinical symptoms, laboratory results, hemodynamic parameters and microbiological analysis in accordance with usual clinical practice, as well as on established criteria for the diagnosis of sepsis: temperature $>38^{\circ} \mathrm{C}$ or $<36^{\circ} \mathrm{C}$, heart rate $>90$ beats per minute, respiratory rate $>20$ breaths per minute, abnormal white blood cell count $>12,000 / \mathrm{mm} 3$ or $<4,000 / \mathrm{mm} 3$ or $>10 \%$ bands, occurrence of hypoperfusion, hypotension and oliguria. (12)

The occurrence of cardiac ischemia and arrhythmias was detected by constant electrocardiographic (ECG) monitoring. Microbiological analysis of bronchial secretions was done every three days in patients included in the study. For patients diagnosed with hospital ventilator associated pneumonia, the frequency of performing microbiological analysis of the bronchial secretion depended on the microbiological lab results and clinical disease course. The dynamics of carrying out hematological, biochemical and other microbiological analysis was determined by the clinical condition of the patient.

Microbiological analyses were performed at the Institute of Microbiology and Molecular Diagnostics of the University Clinical Hospital Mostar in a way that pathogens were isolated and identified from the samples along with determining their potential resistance to certain antibiotics according to the antibiogram. Hematological and biochemical results were collected at the Institute of Laboratory Diagnostics of the University Clinical Hospital Mostar. The following criteria were noted for every patient in both groups: the number of days on mechanical ventilation, the total number of ICU days, and the treatment outcome.

The study reported difficulties in weaning the patients from the ventilator and incidences of re-intubation of patients. Bronchoscopic examination of the larynx and trachea was performed within 1 to 5 days of removal of the cannula or endotracheal tube and any probable functional or anatomical injuries were recorded. We also noted clinical symptoms of dysphagia, dysphonia, dyspnea, etc. Data were collected by the physicians from the Department of Anesthesiology and Reanimation and Intensive Care of the University Clinical Hospital Mostar, while percutaneous tracheostomy was performed by the same and experienced ICU attending physician.

A software system SPSS forWindows(v ersion17.0,SPSSInc., Chicago, Illinois, USA) and MicrosoftExcel (version Office 2007,Redmond,WA,USA) was used for statistical analysis of the obtained data.

Results of nominal characteristics are expressed as n (\%), Chi-square test was used to test significant differences (if lack of expected frequency occurred the Fisher's exact test was used). Kolmogorov-Smirnov test was used to test concurrency of interval characteristics with normal distribution. Characteristics which did not deviate from the normal distribution are expressed as mean and standard deviation $(\mathrm{M} \pm \mathrm{SD})$, while $\mathrm{t}$-Test was used to test significance of the difference between the means of two independent samples. Characteristics which deviated from the normal distribution are expressed as median and interquartile range (C [IQ]) while MannWhitney test was used to test significance of the difference. The level of significance was set at 0.050 while values which could not be expressed to three decimal places are reported as $\mathrm{p}<0.001$.

\section{RESULTS}

Distribution of patients according to tracheotomy and other analyzed parameters ispresented in Table 1.

There was no statistically significant difference between the observed groups in regards to gender $(\mathrm{p}=0.148)$ and median age $(\mathrm{p}=0.386)$ and APACHE II score $(\mathrm{p}=0.472)$. There wasno statistically significant difference between the groups in the distribution of patients by predicted mortality rates according to APACHE II score $(\mathrm{p}=0.187)$.

The difference found in the mean value 
of the SOFA score between the observed groups is not statistically significant $(\mathrm{p}=0.213)$.

The difference found in the incidence of pneumonia among patients from the observed groups is not statistically significant $(\mathrm{p}=0.128)$, but patients who underwent LT were found to be more likely to develop pneumonia than those who underwent ET (OR=2.000; 95\% CI: 0.816-4.902).

Sepsis is more likely to occur in patients who had LT than in patients who had ET $(\mathrm{OR}=2.479 ; 95 \% \mathrm{CI}: 0.995-6.176)$. The difference found is statistically significant $(\mathrm{p}=0.049)$.

The ET group showed less mortality, the difference is statistically significant $(\mathrm{p}=0.028)$.

According to APACHE II score values, $57.9 \%$ of patients who had ET are in the third mortality group. Distribution of patients by group and outcome shows that the portion of patients who died is $44.7 \%$ in the group of patients who underwent ET and $69.0 \%$ in the group of patients who underwent LT. The actual mortality is much higher in the group of patients who had LT. Length of ICU stay and duration of mechanical ventilation according to the groups are presented in Table 2.

The patients who had LT stayed in the ICU longer, and the difference found in the median length of ICU stay between the observed groups is statistically significant $(\mathrm{p}<0.001)$.

Patients who had LT were on mechanical ventilation longer, and the difference found in the median duration of mechanical ventilation between the observed groups is statistically significant $(\mathrm{p}<0.001)$. Adverse events associated with tracheotomy are indicated in Table 3.

Only one patient $(2.38 \%)$ in the late tracheotomy group experienced an adverse event.

\section{DISCUSSION}

The benefits of tracheotomy include: improved patient comfort due to reduction of oropharyngeal and laryngeal stimulation (and possibly less damage to the larynx), less need for sedation, improvement of pulmonary toilet, lower work of breathing, faster weaning from mechanical ventilation, lower risk of ventilator-associated pneumonia, and perhaps shorter duration of mechanical ventilation. By preventing microaspiration of secretions, tracheotomy might reduce VAP and septic episodes. Based on available data, we believe it is reasonable to perform early tracheotomy

Table 1. Distribution of patients according to the type of tracheotomy and analyzed parameters

\begin{tabular}{lllll}
\hline & Tracheostomy & Early $(\mathrm{ET})$ & Late $(\mathrm{LT})$ & $\mathrm{p}$ \\
\hline Gender & $\mathrm{n}$ & 38 & 42 & \\
& Men & $30(78,9.0)$ & $27(64.3)$ & $0.148^{\star}$ \\
& Women & $8(21.1)$ & $15(35.7)$ & \\
\hline Age & $\mathrm{C}[\mathrm{IQ}]$ & $60.0[13.0]$ & $61.5[28.0]$ & $0.386 \ddagger$ \\
\hline APACHE II & $0-9(0 \%)$ & $3(7.9)$ & $1(2.4)$ & $0.187 \dagger$ \\
& $10-19(22 \%)$ & $6(15.8)$ & $15(35.7)$ & \\
& $20-29(58 \%)$ & $22(57.9)$ & $20(47.6)$ & \\
\hline SOFA & $\geq 30(90 \%)$ & $7(18.4)$ & $6(14.3)$ & $0.472 \S$ \\
\hline Pneumonia & M \pm SD & $23.61 \pm 8.14$ & $22.36 \pm 7.32$ & $0.213 \S$ \\
& Yes \pm SD & $13.97 \pm 2.65$ & $14.80 \pm 3.17$ & $0.128^{\star}$ \\
\hline Sepsis & No & $18(47.4)$ & $27(64.3)$ & \\
& Yes & $20(52.6)$ & $15(35.7)$ & $0.049^{\star}$ \\
\hline Outcome & No & $18(47.4)$ & $29(69.0)$ & \\
& Transfer to the ward & $21(55.3)$ & $13(31.0)$ & $0.028^{\star}$ \\
& Dead & $17(44.7)$ & $29(69.0)$ &
\end{tabular}

Data are expressed as $\mathrm{n}(\%)$, except where indicated otherwise

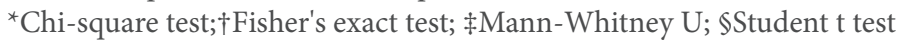

ET, early tracheotomy; LT, late tracheotomy.

Table 2. Length of ICU (intensive care unit) stay and duration of mechanical ventilation according to group

\begin{tabular}{lllll}
\hline & \multicolumn{2}{l}{ Length of ICU stay } & \multicolumn{2}{l}{ Duration of mechanical ventilation } \\
\hline $\mathrm{ET}$ & LT & ET & LT \\
\hline $\mathrm{Me}$ & 38 & 42 & 38 & 42 \\
\hline $\mathrm{IQ}$ & 15.0 & 29.5 & 13.0 & 20.0 \\
\hline Min & 12.0 & 21.0 & 12.0 & 18.0 \\
\hline Max & 5.0 & 4.0 & 3.0 & 4.0 \\
\hline Range & 133.0 & 130. & 110.0 & 110.0 \\
\hline & 128.0 & 126.0 & 107.0 & 106.0 \\
\hline
\end{tabular}

${ }^{*}$ Mann-Whitney U

ET, early tracheotomy; LT, late tracheotomy

Table 3. Potential adverse side-effects associated with percutaneous tracheotomy

\begin{tabular}{llll}
\hline & & ET & LT \\
\hline Intraoperative & Minor bleeding & 38 & 42 \\
\hline Postoperative & Stoma inflammation & 0 & $1(2.38)$ \\
\hline & Subcutaneous emphysema & 0 & 0 \\
\hline & Total & 0 & 0 \\
\hline
\end{tabular}

ET, early tracheotomy; LT, late tracheotomy

in all patients who require prolonged mechanical ventilation.

In most studies and meta analyses a common limitation is heterogeneity of studied populations, as well as methodological variability of the study population, such as time of ET, inclusion and exclusion criteria, tracheotomy techniques etc.

Defining and predicting the need for prolonged ventilation has been a major methodological problem and challenge. The first question that arises is the follow- 
ing: is it possible to prospectively identify patients who will require prolonged mechanical ventilation and could they potentially benefit from tracheotomy? Most physicians base their decision to perform tracheotomy on subjective clinical criteria. Seneff et al. attempted to develop a prediction equation for a large population of mixed ICU patients. Using APACHE III score data from 6,000 ICU patients from 40 United States' hospitals the authors conducted multivariate regression analysis to determine if particular patient or disease characteristics on ICU day one could be used to determine the duration of mechanical ventilation. They found that the admitting diagnosis and degree of physiological derangement measured by APACHE III score could accurately predict duration of mechanical ventilation (MV) in a group of ICU patients. (13)

Heffner et al. developed an acute respiratory distress syndrome (ARDS) predictive score. Predictor factors incorporated in the ARDS Score were collected on Day 4 and Day 7 in 50 patients with ARDS in critical care units of a large Academic Medical Center over a 4 -year period. ARDS Score threshold of $\geq 2.5$ accurately identified patients at risk of prolonged mechanical ventilation. (14)

Troche et al. confirmed that only emergency intubation and a lung injury score greater or equal to 1 independently predicted a duration of mechanical ventilation of more than 14 days. (15)

Terragni et al. estimated the need for prolonged mechanical ventilation according to objective criteria such as the severity of illness Simplified Acute Physiology Score (SAPS II) and SOFA score and the need for ventilator support required to obtain predefining oxygenation criteria. (16)

Most studies included patients who were expected to require prolonged mechanical ventilation based on the clinical assessment by the physician. (17)

In our study we proposed an early tracheotomy decision algorithm based on objective criteria: APACHE II score $>10$, SOFA score $>5$ and a worsening of respiratory parameters as a valuable tool that my help us to predict the duration of mechanical ventilation in a select population of surgical patients.

The ideal time for performing tracheotomy remains controversial. The best timing of tracheotomy has changed over the last several years and now many authors suggest performing early tracheotomy within $2-7$ days. (18)

Complications of prolonged endotracheal intubation include: dental damage, sinus obstruction, sinus infection, mouth injury, laryngeal trauma, cricoid cartilage damage, tracheal injury (from cuff and tube tip). In addition to the other benefits of tracheostomy, the connection between the risk of larynotracheal injury and duration of intubation is important to take into consideration in the timing of tracheostomy. Direct airway examination demonstrates marked airway changes within several days of translaringeal intubation. (18)

In our study, early tracheotomy was performed within 2-4 days of mechanical ventilation to avoid the above mentioned complications.

We have observed a large difference in the incidence of pneumonia: $46.7 \%$ in the ET group versus $64.3 \%$ in the LT group but without statistical significance $(\mathrm{p}=0,056)$. In their systematic review and meta-analysis, Liu et al. reported the crude cumulative incidence of pneumonia as $35.9 \%$ in the ET group and $45.5 \%$ in the LT group. A pooled analysis was not performed secondary to significant heterogeneity of the measured effects between individual studies. (19)

Nseir et al. conducted a retrospective case - control study matching 177 patients who underwent tracheotomy with 177 control subjects treated with prolonged TLI. They found a statistically significant reduction in VAP episodes per 1000 mechanical ventilator days. The multivariate analysis of the study demonstrated that tracheotomy is independently associated with decreased risk for ventilator-associated pneumonia. (20)

The study by Rumbak et al. reported significantly less pneumonia incidences (5\% vs.25\%) in the two groups of observed critically ill medical patients. (9) Zheng et al. report reduced incidences of ventilator associated pneumonia next to the above mentioned. (5)

Contrary to the above, Teragini et al. conducted a randomized control study in 12 ICUs and compared the incidences of hospital pneumonia in patients who underwent ET (6-8 days after translaryngeal intubation) and in those patients who underwent LT (15 days after translaryngeal intubation), and found no statistically significant difference. (16)

In a study conducted by Blot et al., the incidence of hospital infection and the number of septic episodes did not statistically differ between the early tracheotomy group of patients and the one that underwent translaryngeal intubation. Greater comfort was the sole benefit afforded by tracheotomy. (21)

Our study, like almost all other, reported a statistically significant shorter duration of mechanical ventilation and length of ICU stay in the ET group (11.00 and 13 days) versus LT group (20.00 and 29.5 days).

Liu et al. reported that the average duration of mechanical ventilation was 17 days for the ET group and 24 days for the LT group. The study results demonstrated that ET is significantly associated with shorter a ICU stay of 9.13 days. (19)

Bourdeka et al. investigated if ET (fifth day) reduces duration of mechanical ventilation. The study reported shorter mechanical ventilation in ET group. After pneumonia was diagnosed, mechanical ventilator time was $6 \pm 4.7$ days for ET group versus $11.7 \pm 6.7$ days for patient endotrachealy intubated or LT group. (22)

Rodriguez et al. prospectively randomized patients to receive either an ET (within 7 days) or LT (greater than 7 days). ET was associated with shorter ICU and hospital stay, and reduction in duration of mechanical ventilation with a mean difference of - 20 days (95\% CI: 20.8 to $-19.2(\mathrm{p}<0.05)$. (23)

Rumbak et al. also found a decreased length of mechanical ventilation with a mean deference of -9.8 days (95\% CI: -11.5 to $-8.12, \mathrm{p}<0.001)$. The ET group spent less time in the intensive care unit (4.8 \pm 1.4 vs. $16.2 \pm 3.8$ days) and on mechanical ventilation $(7.6 \pm 2.0$ vs. $17.4 \pm 5.3$ days). The generalizability of this study is limited by inclusion of only a very specific group of severely ill medical patients. (9) Griffiths and al. analyzed 5 trials in which ET was performed within 1-7 of mechanical ventilation or ICU admission. The study participants included general trauma, head trauma, surgical, medical and burn patients. The meta-analysis demonstrated faster weaning and shorter duration of mechanical ventilation and ICU stay. (24)

Young and al. did not find a significant reduction in the duration of mechanical ventilation with a mean difference of 1.6 days. (25)

The effect of tracheotomy on intensive care unit, hospital and overall mortality is also controversial.

In most published studies mortality was defined as hospital mortality. The definition included usually ICU, 28 and 30 day mortality and hospital mortality. Hospital mortality of our patients was $46.70 \%$ in ET and $69.00 \%$ in the LT group. The main cause of death was sepsis $43.30 \%$ in ET group and $69.00 \%$ in LT group.

Liu and al. reported mortality data from eight studies. The percentage of patients who died in the early tracheotomy group was $30.7 \%$ compared with $34.7 \%$ in the late 
tracheotomy group. The weighted absolute and relative risk reduction was not significant. Soubgroup analysis did not suggest a difference in outcome based on etiology of critical illness or whether the ET was performed at 2 to 3 days, 4 to 5 days or 7 to 8 days of endotracheal intubation. (19)

A prospective, randomized study by Blot et al. found no difference for a 28 - day mortality in 123 patients included in the study $20 \%$ in ET group and 24\% in LT group. (21) Two single center studies by Koch et al. and Zheng et al. also did not show a survival benefit for ET. In a multicenter nonrandomized, propensity matched comparison, Clech et al. found no difference in critical care unit mortality. $(6,5,26)$

A further study by Scales et al. performed a stratified propensity score analysis from a local database and reported a small increase in long term mortality in the ET, but the increase was of questionable clinical significance. (27)

Prospective and randomized study by Rumbak et al. included 120 medical patients in the ICUs. Patients were critically ill with APACHE II score $>25.50 \%$ were chronic obstructive pulmonary disease (COPD) patients. ET was performed within 2 days of admission while LT was done at days 14-16. In that study the projected need for prolonged intubation was indicated as specific inclusion criteria, but Rumbak did not describe the method by which these patients were identified. Early group showed significantly less mortality ( $31.7 \%$ vs. $61.7 \%)$. The generalizability of this study is limited by inclusion of only a very specific group of severely ill medical patients. (9)

The meta analysis of Dunham et al. included 5 studies of high-quality design. Early tracheotomy was performed within 5 days from the start of mechanical ventilation in a group of trauma patients. There was no statistically significant difference in the risk of developing pneumonia and duration of mechanical ventilation but mortality was significantly lower and ICU stay significantly shorter in ET group. (28) Frutos Vivar et al. found lower ICU mortality rate in patients undergoing tracheotomy (20\% vs. $32 \%$ ) but hospital mortality was not different between the groups. (29) Wang et al. in a systematic review and meta-analysis of seven randomized control trials with 1044 patients concluded that the timing of tracheotomy did not significantly alter important clinical outcomes of the patients-short and long term mortality and incidence of ventilator -associated pneumonia. (30)

Most of the meta analysis and RCT trials published on ET have found no important benefits with ET. There was no difference in hospital mortality and there was not enough evidence to support ET with regard to the incidence of VAP. In most stud- ies ET was significantly associated with a decreased length of ICU and hospital stay and length of mechanical ventilation. The literature on this topic is difficult to interpret because of varying definition of early tracheotomy 0 to 7 days and inhomogeneous groups of patients, different inclusion and exclusion criteria, randomization techniques, different weaning protocols and because some of the studies are retrospective.

The most important limitation of our study is the small number of included patients, leading to underpowered (unsupported) results. In addition, the study is limited by being a single - center study.

On the other hand, we believe that our study is free of the main methodological problems. We have a homogenous population in terms of pathology and in terms of severity of illness because we used objective inclusion criteria in the study populations and we precisely defined the timing for performing ET.

The results of our study support ET performed $2-4$ days from the start of mechanical ventilation in regards to the length of mechanical ventilation and length of ICU stay. Despite the lack of power, we found significant benefits of early tracheotomy regarding the incidence of pneumonia, sepsis and hospital mortality.

\section{REFERENCES}

1. Rogers JM. Intubation vs. Tracheotomy. Can Med Assoc J. 1922;12:238-40.

2. Durbin CG. Tracheostomy: Why, when, and how? Respiratory Care 2010;55:1056-8.

3. Cox CE, Carson SS, Holmes GM, Howard A, Carey TS. Increase in tracheostomy for prolonged mechanical ventilation in North Carolina, 1993-2002. Crit Care Med 2004;32:2219-26.

4. Adesina O, Soufi S, Alhamad M, Walsmann G, Shilbi M. Early Versus Late Tracheostomy in Critically Ill Patients. Chest [serial online] October 2011 [Cited 2017 May 17]. Available from: URL:http://journal.publications.chestnet.org/article.aspx?articleid=1045937

5. Zheng Y, Sui F, Chen XK, Zhang GC, Wang XW, Zhao S, et al. Early versus late percutaneous dilational tracheostomy in critically ill patients anticipated requiring prolonged mechanical ventilation. Chin Med J (Engl) 2012;125:1925-30.

6. Koch T, Hecker B, Hecker A, Brenck F, Preuß M, Schmelzer T, et al. Early tracheostomy decreases ventilation time but has no impact on mortality of intensive care patients: a randomized study. Langenbecks Arch Surg 2012;397:1001-8.

7. Ahmed N, Kuo YH. Early versus late tracheostomy in patients with severe traumatic head injury. Surg Infect (Larchmt) 2007;8:343-7.

8. Mahafza T, Batarseh S, Bsoul N, Massad E, Qudaisat I, Al- Layla AE. Early vs. late tracheostomy for the ICU patients: Experience in a referral hospital. Saudi J Anaesth 2012;6:152-4

9. Rumbak MJ, Newton M, Truncale T, Schwartz SW, Adams JW, Hazard PB. A prospective, randomized, study comparing early percutaneous dilational tracheotomy to prolonged translaryngeal intubation (delayed tracheotomy) in critically ill medical patients. Crit Care Med 2004;32:1689-94.

10. Brook AD, Sherman G, Malen J, Kollef MH. Early versus late tracheostomy in patients who require prolonged mechanical ventilation. Am J Crit Care. 2000;9:352-9.

11. American Thoracic Society, Infectious Diseases Society of America. Guidelines for the management of adults with hospital-acquired, ventilator-associated, and healthcare-associated pneumonia. Am J Respir Crit Care Med 2005;171:388-416.

12. Levy MM, Fink MP, Marshall JC, Abraham E, Angus D, Cook D, et al. 2001 SCCM/ESICM/ACCP/ATS/SIS International Sepsis Definitions Conference. Intensive Care Med 2003;29:530-8.

13. Seneff MG, Zimmerman JE, Knaus WA, Wagner DP, Draper EA. Predicting the duration of mechanical ventilation. The importance of disease and patient characteristics. Chest 1996;110:469-76.

14. Heffner JE, Brown LK, Barbieri CA, Harpel KS, DeLeo J. Prospective validation of an acute respiratory distress syndrome predictive 
score. Am J Respir Crit Care Med 1995;152:1518-25.

15. Troche G, Moine P. Is the duration of mechanical ventilation predictable? Chest 1997;112:745-51.

16. Terragni PP, Antonelli M, Fumagalli R, Faggiano C, Berardino M, Pallavicini FB, et al. Early vs late tracheotomy for prevention of pneumonia in mechanically ventilated adult ICU patients: a randomized controlled trial. JAMA 2010;303:1483-9.

17. Diaz-Prieto A, Mateu A, Gorriz M, Ortiga B, Truchero C, Sampietro N, et al. A randomized clinical trial for the timing of tracheotomy in critically ill patients: factor precluding inclusion in a single center study. Crit Care 2014;18:585-99.

18. Durbin CG Jr. Early complications of tracheostomy. Respir Care 2005;50:511-5.

19. Liu CC, Livingstone ED, Dort JC. Early versus late tracheostomy: a systematic review and meta-analysis. Otolaryngology-Head AND Neck Surgery 2015;152:219-27.

20. Nseir S, Di Pompeo C, Jozefowicz E, Cavestri B, Brisson H, Nyunga M, et al. Relatioship between tracheotomy and ventilator -associated pneumonia: a case control study. Eur Respir J 2007;30:314-20.

21. Blot F, Similowski T, Trouillet JL, Chardon P, Korach JM, Costa MA, et al. Early tracheotomy versus prolonged endotracheal intubation in unselected severely ill ICU patients. Intensive Care Med 2008;34:1779-87.

22. Bouderka MA, Fakhir B, Bouaggad A, Hmamouchi B, Hamoudi D, Harti A. Early tracheostomy versus prolonged endotracheal intubation in severe head injury. J Trauma 2004;57:251-4.

23. Rodriguez JL, Steinberg SM, Luchetti FA, Gibbons KJ, Taheri PA, Flint LM. Early tracheostomy for primary airway management in the surgical critical care setting. Surgery 1990;108:655-9.

24. Griffiths J, Barber VS, Morgan L, Young JD. Systematic review and meta-analysis of studies of the timing of tracheostomy in adult patients undergoing artificial ventilation. BMJ 2005;330:1243.

25. Young D, Harrison DA, Cuthbertson BH, Rowan K; TracMan Collaborators. Effect of early versus late tracheotomy placement on survival in patients receiving mechanical ventilation. JAMA 2013;309:2121-9.

26. Clec'h C, Alberti C, Vincent F, Garrouste-Orgeas M, de Lassence A, Toledano D, et al. Tracheotomy does not improve the outcome of patients requiring prolonged mechanical ventilation: a propensity a propensity analysis. Crit Care Med 2007;35:132-8.

27. Scales DC, Thiruchelvam D, Kiss A, Redelmeier DA. The effect of trachostomy timing during critical care illness on long term survival. Crit Care Med 2008;36:2547-57.

28. Dunham CM, Ransom KJ. Assesment of early tracheostomy in trauma patients: a systematic review and meta -analysis. Am Surg 2006;72:276-81.

29. Frutos-Vivar F, Esteban A, Apezteguía C, Anzueto A, Nightingale P, González M, et al. Outcome of mechanically ventilated patients who required tracheostomy. Crit Care Med 2005;33:290-8.

30. Wang F, Wu Y, Bo L, Lou J, Zhu J, Chen F, et al. The timing of tracheotomy in critically ill patients undergoing mechanical ventilation: a systematic review and meta-analysis of randomized controlled trials. Chest. 2011;140:1456-65. 